\title{
Photoacoustic spectroscopy of $\beta$-hematin
}

\author{
Edward B. Samson ${ }^{1,2}$, Benjamin S. Goldschmidt ${ }^{1,2}$, Paul J. D. Whiteside ${ }^{1,2}$, Amanda S. M. \\ Sudduth $^{1,2}$, John R. Custer ${ }^{1,2}$, Brenda Beerntsen ${ }^{3}$, and John A. Viator ${ }^{1,2,4}$ \\ John A. Viator: viatorj@missouri.edu \\ ${ }^{1}$ Department of Biological Engineering, University of Missouri-Columbia, Columbia, Missouri, \\ 65211, USA \\ ${ }^{2}$ Christopher S. Bond Life Sciences Center, University of Missouri-Columbia, Columbia, Missouri, \\ 65211, USA \\ ${ }^{3}$ Department of Animal Sciences, University of Missouri-Columbia, Columbia, Missouri, 65211, \\ USA \\ ${ }^{4}$ Department of Dermatology, University of Missouri-Columbia, Columbia, Missouri, 65211, USA
}

\begin{abstract}
Malaria affects over 200 million individuals annually, resulting in 800,000 fatalities. Current tests use blood smears and can only detect the disease when $0.1-1 \%$ of blood cells are infected. We are investigating the use of photoacoustic flowmetry to sense as few as one infected cell among 10 million or more normal blood cells, thus diagnosing infection before patients become symptomatic. Photoacoustic flowmetry is similar to conventional flow cytometry, except that rare cells are targeted by nanosecond laser pulses to induce ultrasonic responses. This system has been used to detect single melanoma cells in $10 \mathrm{ml}$ of blood. Our objective is to apply photoacoustic flowmetry to detection of the malaria pigment hemozoin, which is a byproduct of parasitedigested hemoglobin in the blood. However, hemozoin is difficult to purify in quantities greater than a milligram, so a synthetic analog, known as $\beta$-hematin was derived from porcine haemin. The specific purpose of this study is to establish the efficacy of using $\beta$-hematin, rather than hemozoin, for photoacoustic measurements. We characterized $\beta$-hematin using UV-vis spectroscopy, TEM, and FTIR, then tested the effects of laser irradiation on the synthetic product. We finally determined its absorption spectrum using photoacoustic excitation. UV-vis spectroscopy verified that $\beta$-hematin was distinctly different from its precursor. TEM analysis confirmed its previously established nanorod shape, and comparison of the FTIR results with published spectroscopy data showed that our product had the distinctive absorbance peaks at 1661 and $1206 \mathrm{~cm}^{-1}$. Also, our research indicated that prolonged irradiation dramatically alters the physical and optical properties of the $\beta$-hematin, resulting in increased absorption at shorter wavelengths. Nevertheless, the photoacoustic absorption spectrum mimicked that generated by UV-vis spectroscopy, which confirms the accuracy of the photoacoustic method and strongly suggests that photoacoustic flowmetry may be used as a tool for diagnosis of malaria infection.
\end{abstract}

\section{Keywords}

Photoacoustics; malaria; hemozoin; $\beta$-hematin; UV-vis spectroscopy 


\section{Introduction}

\subsection{Background}

Contrary to popular belief, despite its prevalence in many tropical climates, malaria is a particularly deadly disease whose vectors are present on every continent barring Antarctica; moreover, there are cases documented each year in even the most temperate and borderline polar climates. In point of fact, the World Health Organization (WHO) estimates that malaria is responsible for over 800,000 deaths annually worldwide [1]. Accordingly, in order to reduce the death toll, the WHO recommends that all individuals suspected to have malaria receive at least one malaria-specific test to confirm or refute the diagnosis, given the lethal nature of the disease if left untreated. However, current microscopy tests and immunoassays are limited in either sensitivity, specificity, or practicality and as such are often ineffective or simply unavailable for confirming most cases of malaria [2,3]. Furthermore, even if correctly diagnosed, the ever-changing nature of malaria renders the disease decidedly difficult to treat due to the rapid proliferation and drug resistance of the parasites. That being the case, the early detection of malarial infection while the concentration of infected erythrocytes is still minimal would enable faster treatment and likely dramatically increase the odds of recovery and reduce the duration of the infection.

Consequently, many more contemporary methods are being researched in hopes of designing a diagnostic tool that is significantly more sensitive than current methods. One alternative to microscopy and immunoassays involves the detection of the malaria-specific pigment hemozoin, a byproduct of hemoglobin digestion by malaria parasites inside host erythrocytes, which can be found freely floating in the bloodstream or inside erythrocytes and leukocytes [4,5]. While hemozoin is not the only biomarker of malarial infection, it is, however, created by all known species of malaria parasites and as such shows promise for use in a truly universal test for malarial infection, most notably because hemozoin is present in leukocytes even before symptoms arise in the patient [6]. Moreover, it is an ideal analyte for many optically based detection modalities, because it naturally strongly absorbs light in the visible spectrum and also because the percentage of leukocytes with the hemozoin pigment has been shown to be directly related to the severity of the infection $[7,8]$.

Unfortunately, the process of deriving and isolating hemozoin from infected cell cultures is laborious, lengthy, and low-yielding, three aspects that are particularly prohibitive of malaria research [9]. Accordingly, a synthetic analog for the malaria pigment, called $\beta$ hematin, has been developed for use as a potential substitute in optical detection methods. The hemozoin substitute is derived from porcine haemin by precipitation in a sodium acetate buffer, which allows for synthesis of $\beta$-hematin at a significantly higher yield and at a fraction of the cost of isolating naturally occurring hemozoin [10]. $\beta$-hematin has also already been used extensively in place of hemozoin in anti-malarial studies in attempts to determine methods to inhibit the digestion of hemoglobin to form hemozoin [11, 12]. Furthermore, once synthesized, the physical and optical properties of $\beta$-hematin can be readily matched to those of hemozoin through Transmission Electron Microscopy (TEM), UV-visible light spectroscopy (UV-vis spectroscopy), and Fourier Transformed Infrared Spectroscopy (FTIR) since prior research has already characterized the distinct properties of purified hemozoin $[6,7,10]$. Considering that the optical properties of hemozoin and $\beta$ hematin have already been determined, it is reasonable to consider previously proven detection methods like photoacoustic spectroscopy for use in the development of an optical detection system for malarial infection that may be up to three or four orders of magnitude more sensitive than currently employed tests.

Essentially, photoacoustic spectroscopy involves the analysis of ultrasonic waveforms generated through the rapid thermoelastic expansion of a medium following the optical 
absorption of pulsed laser light [13]. More specifically, because the waveforms are generated based on the optical properties of the absorber, they are unique to the absorber and may therefore be used for characterization and detection. Provided the laser energy is completely absorbed quickly enough to prevent dissipation in the form of pressure, a condition known as stress confinement, the thermoelastic expansion will result in an ultrasonic pressure wave defined by the spatial pressure distribution:

$$
P(z)=\mu_{e f f} \Gamma H_{0} e^{-\mu_{e f f} z}
$$

where $\mu_{\text {eff }}$ is the effective optical absorption coefficient, $\Gamma$ is the unitless Grüneisen coefficient, $H_{0}$ is the incident laser radiant exposure, and $z$ is the depth of the acoustic wave in the medium [14]. For the remainder of this paper, however, we will consider that the optically scattering component of $\mu_{\text {eff }}$ is negligible, and so $\mu_{\text {eff }}$ will be replaced with the specific optical absorption coefficient $\mu_{a}$. Acting on this principle, photoacoustic systems have been utilized in a myriad of applications including the observation of refractive index changes as a result of photoacoustically generated pressure waves by Paltauf et al. [15] and in the development of an evanescent wave optical detection modality making use of total internal reflection photoacoustic spectroscopy (TIRPAS) being developed by Sudduth et al. [16]. Moreover, photoacoustics has also been used to detect circulating melanoma cells by Weight et al. [17], gold nanorod tagged prostate cancer cells by Viator et al. [18], and even to distinguish between viable and coagulated blood in burn victims by Talbert et al. [19], so it seems only prudent to apply this technology to the detection of $\beta$-hematin given its highly absorbing nature, even in relatively low concentrations.

\subsection{Objectives}

In order to initiate the development of a malaria-specific photoacoustic detection assay, it must first be established that photoacoustics is a viable method for the detection of $\beta$ hematin, and therefore of hemozoin. However, given the limited availability of $\beta$-hematin in sufficient quantities for research applications, the conditions of this project necessitated that we derive the hemozoin analog in house using methods previously established by Egan et al. [10]. Moreover, the effects of laser irradiation on $\beta$-hematin has yet to be conclusively demonstrated, which may inhibit attempts to use optical detection modalities. Consequently, this study serves as a pilot to ascertain the efficacy of a photoacoustic detection method for $\beta$-hematin and hemozoin, and subsequently to justify the continued development of a photoacoustic replacement for the inadequate contemporary tests for malarial infection.

Therefore, the objectives of this study were as follows:

- to verify that the procedures chosen to derive $\beta$-hematin produce an appropriate analog for hemozoin with regard to both physical and optical properties via TEM, FTIR, and UV-vis spectroscopy

- to observe any effects that prolonged laser irradiation may have on the optical and physical characteristics of $\beta$-hematin

- to obtain an optical absorption spectrum of $\beta$-hematin via the least-squares curve fitting of photoacoustic waveforms for comparison with an absorption spectrum obtained using UV-vis spectroscopy to confirm the accuracy of the photoacoustic method and verify its viability as a detection modality worthy of further study. 


\section{Materials and Methods}

\section{1. $\beta$-hematin synthesis}

The synthesis of $\beta$-hematin began by slowly dissolving $40 \mathrm{~g}$ of $\mathrm{NaOH}$ pellets into a $150 \mathrm{ml}$ beaker containing $50 \mathrm{ml}$ of rapidly stirred distilled water to create a $12.9 \mathrm{M} \mathrm{NaOH}$ solution, while making sure to cover the beaker to prevent evaporation. Meanwhile, $75 \mathrm{ml}$ of $17.1 \mathrm{M}$ glacial acetic acid was added to a second $150 \mathrm{ml}$ beaker. While rapidly stirring the glacial acetic acid with a magnetic stir bar, the $12.9 \mathrm{M} \mathrm{NaOH}$ solution was added drop-wise until the $\mathrm{pH}$ of the titrated buffer solution was approximately 4.5 , at which point distilled water was added until the total solution volume was $100 \mathrm{ml}$. Then $34 \mathrm{ml}$ of $0.1 \mathrm{M} \mathrm{NaOH}$ solution was heated in a $50 \mathrm{ml}$ beaker to $60^{\circ} \mathrm{C}$ using a hot water bath. When the solution reached the appropriate temperature, $175.5 \mathrm{mg}$ of haemin (porcine haemin, Sigma Aldrich, St. Louis, Missouri) was dissolved while stirring constantly and covering the beaker to avoid solvent loss from evaporation. Then the haemin solution was neutralized with $3.4 \mathrm{ml}$ of $1 \mathrm{M} \mathrm{HCl}$ solution.

In a separate, covered $100 \mathrm{ml}$ beaker, $20.69 \mathrm{ml}$ of the prepared acetic acid buffer was heated in a hot water bath to $60^{\circ} \mathrm{C}$. After 3-5 minutes with continued stirring, the $20.69 \mathrm{ml}$ of acetic acid buffer was added to the neutralized haemin solution. The resulting solution was covered and stirred vigorously at a constant temperature of $60^{\circ} \mathrm{C}$ for 2 hours, after which point it was put in an ice bath for 10 minutes. The cooled solution was then filtered over $8.0 \mu \mathrm{m}$ nitrocellulose filter paper (Millipore, Bellerica, Massechusetts). The filtered precipitate was then washed with distilled water and allowed to dry for 5 minutes. Finally, the solid was collected and dried in a desiccator containing a silica gel drying agent for 48 hours, and subsequenlty was stored away from light exposure at $8^{\circ} \mathrm{C}$.

\subsection{Characterization of $\beta$-hematin}

The physical and optical characterization of $\beta$-hematin consisted of three components: TEM, FTIR, and UV-vis spectroscopy. The TEM images were taken at the Electron Microscopy Core Facility of the University of Missouri. Samples of hemozoin and pristine and irradiated dilute $\beta$-hematin suspensions were placed on separate copper grids, removing the solvent after 5 minutes. The samples were then examined under the electron microscope using standard imaging procedures.

For the UV-vis spectroscopy analysis, an Ocean Optics HR200 High-Resolution Spectrometer was used to determine the absorption spectrum of $\beta$-hematin and that of its porcine precursor, haemin, for comparison. A sample of $1 \mathrm{mg} / \mathrm{ml}$ of $\beta$-hematin dispersed in a phosphate buffered saline (PBS) solution at room temperature was pipetted via capillary action between two $1 \mathrm{~mm}$ thick glass slides separated by two $155 \mu \mathrm{m}$ thick glass cover slips, which served to form an optical pathway of known thickness. An absorption spectrum was recorded using SpectraSuite software, using a PBS reference sample to record light and dark reference spectra. An absorption spectrum of haemin solution was recorded in the same way.

Finally, for FTIR characterization of $\beta$-hematin, approximately $5 \mathrm{mg}$ of solid $\beta$-hematin was ground into a fine powder and packed onto a single-reflection diamond-attenuated total reflectance accessory. The $\beta$-hematin was then scanned from 10,000 to $55 \mathrm{~cm}^{-1}$ using OMNIC software.

\subsection{Irradiated $\beta$-hematin absorption and structure}

In order to examine the effects of laser irradiation on both the physical and optical properties of $\beta$-hematin prior to any photoacoustic analysis, a volume of $20 \mathrm{ml}$ dilute $\beta$-hematin suspended in PBS was mixed in a $50 \mathrm{ml}$ beaker, of which $10 \mathrm{ml}$ was set aside to serve as the 
pristine solution with the remaining $10 \mathrm{ml}$ in a separate $50 \mathrm{ml}$ beaker to be irradiated with 532nm laser light. The 532nm laser light from a Vibrant 355 II Tunable Laser System (Vibrant, Opotek, Carlsbad, California) was coupled into a $1000 \mu \mathrm{m}$ glass optical fiber. The energy out of the fiber prior to irradiation was measured to be $7.32 \mathrm{~mJ}$ and the end of the optical fiber was positioned approximately $5 \mathrm{~mm}$ above the surface of the $\beta$-hematin suspension and $10 \mathrm{~cm}$ from the center of the cylindrical beaker. To observe the prolonged effects of irradiation, the solution was pulsed at $20 \mathrm{~Hz}$ for 10 minutes while being stirred vigorously.

In order to obtain the optical absorption spectra of the pristine and irradiated samples, the samples were placed in a $5 \mathrm{~mm}$ diameter by $1 \mathrm{~mm}$ deep glass cuvette covered by a $155 \mu \mathrm{m}$ glass cover slip. The absorption spectra of the cuvettes were recorded using a spectrometer positioned on its side such that the optical fibers leading to and from the sample holder were vertically oriented, again using a PBS solution for the light and dark reference spectra.

\subsection{Photoacoustic apparatus}

The apparatus constructed for the generation of photoacoustic waveforms made use of the same Vibrant 355 II laser system from the previous section, which consists of a Q-Switched Nd:YAG laser whose output wavelength is tuned by an optical parametric oscillator controlled using OPOTEK Control Software v1.093. The laser light was again coupled into $1000 \mu \mathrm{m}$ glass optical fiber, but as shown in Figure 1, the tip of the fiber was positioned $23.7 \mathrm{~mm}$ above the surface of the cuvette resulting in a $7 \mathrm{~mm}$ diameter disc of incident laser light on the surface of the sample. The cuvette itself was made in house from an acrylic tube $20 \mathrm{~mm}$ in diameter cut to length and sealed on its base by Saran wrap, which when filled with $2 \mathrm{ml}$ of $1 \mathrm{mg} / \mathrm{ml} \beta$-hematin dispersed in PBS resulted in a sample depth of $6 \mathrm{~mm}$. In order to avoid any potentially detrimental effects of laser irradiation, the energy output of the laser was kept under $2 \mathrm{~mJ}$ out of the fiber.

The sample was placed between the optical fiber and the face of an ultrasonic transducer, also made in house. The active element of the piezoelectric transducer was a $25 \mu \mathrm{m}$ Polyvinylidene fluoride (PVDF) film with gold foil on both sides. The negative side of the PVDF faced the sample and was connected to ground, whereas the positive faced away from the sample and was connected to a BNC coaxial port attached to the transducer housing. In order to prevent bleaching the PVDF surface with excess laser light, a $.55 \mathrm{~mm}$ thick acrylamide optically scattering pad was placed between the sample and the transducer face, all of which were acoustically coupled with ultrasound gel. The transducer was connected to the input of an amplifier (RITEC Broadband Receiver BR-640A, Warwick Rhode Island), which was set to $28 d B$ gain with input impedence set to "High" and a high pass filter set to a cutoff frequency of $100 \mathrm{kHz}$. The output of the amplifier was then connected to the input channel of an oscilloscope (Tektronix TDS 2024B, Wilsonville, Oregon) along with a photodiode to trigger the sampling. The waveforms were aquired at $250 \mathrm{~ns}$ per division with a sample interval of $1 \mathrm{~ns}$ and a recording length of 2500 samples.

The properties of the homemade transducer were characterized by photoacoustically illuminating a sample of exceptionally highly absorbing dye solution, which resulted in the generation of a Dirac delta signal. The Fast Fourier Transform of the generated waveform was therefore considered to be the transfer function of the transducer, from which we determined its central frequency to be $28 \mathrm{MHz}$ with a bandwidth of accepted frequencies in the range of 14 to $42 \mathrm{MHz}$. 


\subsection{Photoacoustic absorption spectra}

The photoacoustic waveforms were each generated by a single $5 \mathrm{~ns}$ laser pulse to decrease the chance of over-irradiating and photobleaching the samples. Additionally, the samples were mixed by a pipette between every pulse. Photoacoustic waveforms of $1 \mathrm{mg} / \mathrm{ml}$ of $\beta$ hematin suspended in PBS were collected from $410-660 \mathrm{~nm}$ at $10 \mathrm{~nm}$ increments. A total of five spectra were recorded, with the $\beta$-hematin sample replaced after each complete spectrum. A full spectrum took about an hour to complete apart from preparation time. Finally, the wavelength-specific absorption coefficients were calculated from the acoustic waveforms using a weighted least-squares curve fitting algorithm written in Matlab as derived by Weisstein [20]. The least-squares algorithm must be weighted in order to emphasize the rapidly growing exponential portion of the photoacoustic waveforms, but fits a standard exponential equation of the form:

$$
Y(x)=A e^{B x}
$$

Defining $\mathrm{B}=\mathrm{b}$ and $\mathrm{A}=$ eathe least squares algorithm then minimizes Equation (3) in order to obtain the least-squares coefficients.

$$
\sum_{i=1}^{n} y_{i}\left(\ln y_{i}-a-b x_{i}\right)^{2}
$$

Applying least squares fitting, solving for the exponential coefficient $b$, and comparing with Equation (1) yields:

$$
\mu_{e f f}=b=\frac{\sum_{i=1}^{n}\left(y_{i}\right) \sum_{i=1}^{n}\left(x_{i} y_{i} \ln y_{i}\right)-\sum_{i=1}^{n}\left(x_{i} y_{i}\right) \sum_{i=1}^{n}\left(x_{i} y_{i} \ln y_{i}\right)}{\sum_{i=1}^{n}\left(y_{i}\right) \sum_{i=1}^{n}\left(x_{i}{ }^{2} y_{i}\right)-\left(\sum_{i=1}^{n} x_{i} y_{i}\right)^{2}}
$$

\section{Results}

\subsection{Synthesis of $\beta$-hematin}

In order to confirm the accurate synthesis of $\beta$-hematin, UV-vis spectroscopy measurements of the absorption spectra of the porcine haemin precursor and the $\beta$-hematin precipitate were recorded and are shown in Figure 2. The reaction yielded $120 \mathrm{mg}$ of dried $\beta$-hematin precipitate whose absorbance spectrum is dramatically different than the haemin precursor. Whereas haemin has a relatively constant absorbance across the visible spectrum, $\beta$-hematin on the other hand appears to be highly absorbing at shorter wavelengths with a steady decreasing trend as wavelength increases and a local maximum around $650 \mathrm{~nm}$. This maximum is a Soret band [21], as well as the band at about $450 \mathrm{~nm}$.

\subsection{Comparison of $\beta$-hematin and hemozoin}

The TEM images shown in Figure 3 depict that the synthesized $\beta$-hematin crystals are 100 by $300 \mathrm{~nm}$ nanorods that also experience hydrogen bonding, which corresponds with the description of the hemozoin structures described by Noland et al. [6]. With regard to the FTIR analysis, the two most prominent absorbance peaks of the $\beta$-hematin product are at 1661 and $1206 \mathrm{~cm}^{-1}$, which are relatively close to the peaks at 1663 and $1210 \mathrm{~cm}^{-1}$ in the purified hemozoin FTIR spectrum published by Egan et al. [10], who concluded that the two peaks resulted from the vibrational stretching of the $\mathrm{C}=\mathrm{O}$ and $\mathrm{C}-\mathrm{O}$ components respectively of the carboxylate group attached to the $\mathrm{Fe}$ (III) center. 
The former peak is believed to arise from the $\mathrm{CO}$ stretch of the carboxylate group coordinated to the $\mathrm{Fe}$ (III) center, while the latter arises from the $\mathrm{CO}$ single bond stretch of the same group

\subsection{Irradiated $\beta$-hematin}

In order to determine the effects of prolonged laser exposure on the physical and optical characteristics of the synthesized $\beta$-hematin, TEM and UV-vis spectroscopy were performed on both pristine and irradiated samples. The TEM images shown in Figure 4 show a visibly distinct difference in physical appearance between the pristine and irradiated $\beta$-hematin samples. Furthermore, the UV-vis spectroscopy absorption spectra shown in Figure 5 also depict a change in the optical properties of the synthesized product. While the two samples do not vary significantly for wavelengths above $650 \mathrm{~nm}$, the irradiated $\beta$-hematin displays a significant increase in absorbance between 400 and $500 \mathrm{~nm}$.

\subsection{Photoacoustic absorption spectrum}

Figure 6 shows a sample photoacoustic waveform at 590nm that was subsequently analyzed by the least-squares algorithm from Section 2.5. Compiling all of the waveforms analyzed as such yielded Figure 7, which shows the average absorption coefficients of five samples of $1 \frac{m g}{m l} \beta$-hematin suspension for each wavelength measured (left) alongside the corresponding UV-vis spectroscopy absorption spectrum (right). The average absorption coefficients at each wavelength were then plotted against their UV-vis spectroscopy equivalents to determine if any distinct relationship was evident, as shown in Figure 8. The data was then fit with simple linear least-squares and the equation relating the calculated photoacoustic coefficients to the observed UV-vis spectroscopy coefficients is given by:

$$
\mu_{a}^{P A}(\lambda)=1.1635 \mu_{a}^{V i s}(\lambda)-22.615
$$

where $\mu_{a}^{P A}(\lambda)$ and $\mu_{a}^{V i s}(\lambda)$ are the average absorption coefficients derived from curve-fitting photoacoustic waveforms and from UV-vis spectroscopy absorbance measurements respectively. The coefficient of determination $\left(\mathrm{R}^{2}\right)$ of the linear regression was 0.977 . It should be noted that the variation between the two means of absorption spectroscopy appears to be fairly similar regardless of absorption coefficient; however, the photoacoustic values appear to be consistently vertically shifted downward by $22.615 \mathrm{~cm}^{-1}$, which will be addressed in later sections.

\section{Discussion}

\subsection{Synthesis of $\beta$-hematin}

The large disparity between the absorption spectra of the synthesized $\beta$-hematin product and its porcine precursor shown in Figure 2 provides evidence that a significant chemical change has occurred during the derivation process. Furthermore, the comparison reveals that there is little to no residual precursor remaining in the product to significantly contribute to the observed optical absorption. Although both of the samples were $1 \frac{\mathrm{mg}}{\mathrm{ml}}$ suspensions in PBS, the absorption spectra are vastly different for the majority of visible and near-infrared light. It should be noted that the mass of $\beta$-hematin yielded was only $69 \%$ of the mass of haemin precursor used; however, this discrepancy may be explained as a result of product loss during filtration and the removal of the hydrogen chloride group as haemin is converted to $\beta$-hematin. 


\subsection{Comparison of $\beta$-hematin and hemozoin}

The similarity of the physical and optical properties of the synthesized $\beta$-hematin to the previously established properties of hemozoin supports our assertion that $\beta$-hematin may be a useful analog for hemozoin in photoacoustic research. However, when analyzing the photoacoustic waveforms, the size of the $\beta$-hematin aggregates must be taken into consideration, since larger aggregates will result in waveforms with larger amplitudes. Typically, the amplitude of the waveform is taken into consideration when determining the absorption coefficient; however, since the $\beta$-hematin aggregates are formed from nanorod monomers, surface plasmon resonance may distort the waveforms to a greater degree as the size of the aggregates increases.

Moreover, these large aggregates of $\beta$-hematin prevent the monomers from evenly dispersing, rendering it difficult to create accurate dilutions of $\beta$-hematin suspensions, and furthermore, may not even be able to be engulfed by leukocytes through phagocytosis, which renders preliminary photoacoustic systems somewhat ineffective at distinguishing infected blood samples. Also, it is unlikely that parasite-derived hemozoin occurs naturally in large enough concentrations to form aggregates akin to those shown in Figure 3 so it would be prudent to remove large aggregates from the solutions prior to testing in future experiments. One potential method of separating aggregates of $\beta$-hematin based on size would be to add the $\beta$-hematin suspension to a sample of leukocytes, allow phagocytosis to occur for all aggregates small enough to be engulfed, and finally filter out the suspension though the use of either cytofunnel centrifugation or a separation column, both of which should separate the cell suspension from the excess $\beta$-hematin suspension.

\subsection{Irradiated $\beta$-hematin}

The TEM images in Figure 4 depict a distinct change in the overall physical properties of the $\beta$-hematin monomers after prolonged laser irradiation. Originally, the monomers conform to a well-organized crystalline macrostructure in the form of $100 \mathrm{~nm}$ by $300 \mathrm{~nm}$ nanorods; however, after prolonged laser exposure, there is a visibly discernible degradation of the nanorods that resulted in the formation of irregularly shaped clusters of irradiated $\beta$ hematin. This blatant change in physical appearance coincided with a significant increase in optical absorption in irradiated $\beta$-hematin over the pristine sample, as shown in Figure 5. The plot of absorption spectra for pristine and irradiated samples clearly depicts a significant rise in absorption at shorter wavelengths of visible light, though after approximately $650 \mathrm{~nm}$ the two samples have comparable absorption. While not included in this study, if this phenomenon were to occur at low concentrations of $\beta$-hematin, it is possible that purposeful alteration of absorption via laser irradiation may provide means by which to significantly increase the photoacoustic signals generated, thereby increasing the sensitivity of a photoacoustic-based detection system. However, it should also be considered that hemozoin may not be affected in exactly the same manner as its synthetic counterpart, so further study is needed to determine the efficacy of intentional over-irradiation for detection purposes.

\subsection{Photoacoustic absorption spectrum}

According to the regression shown in Figure 8 the relationship between the photoacoustically derived absorption coefficients and those determined by UV-vis spectroscopy is approximately linear with a slope near one and minimal variation (Equation 5), which suggests that the two spectra are very similar in shape. However, there is a significant vertical shift signifying that the photoacoustic values are consistently lower than those from UV-vis spectroscopy by at least one standard deviation. Furthermore, as previously mentioned, $\beta$-hematin was originally assumed to have insignificant optical scattering properties for the calculation of the absorption coefficient; however, the 
discrepancy between the two spectroscopy methods may indicate that there is more scattering than initially anticipated.

That disparity notwithstanding, it is reasonable to conclude that the large spot size and relatively low energy of the incident laser beam in conjunction with sample-mixing between pulses effectively prevented over-irradiation from altering the optical properties of the samples. The aforementioned irradiation study covered by Figure 5 suggests that since overirradiation of $\beta$-hematin drastically increases the absorption at shorter wavelengths, any significant damage to the suspension would have presented as a noticeable transformation of the shape of the photoacoustic absorption spectrum as opposed to a simple shift in magnitude.

\section{Conclusion}

Based on the TEM, FTIR, and UV-vis spectroscopy results, the process chosen to synthesize $\beta$-hematin appears to produce a viable replacement for hemozoin in optical detection methods including, but not limited to, photoacoustic spectroscopy. Furthermore, the photoacoustically determined absorption spectrum of $\beta$-hematin essentially mirrored the shape of that obtained by UV-vis spectroscopy, which verifies the efficacy of using photoacoustics as an accurate optical characterization technique, provided the shift in magnitude can be resolved either computationally or by redesigning the photoacoustic apparatus.

However, due to the drastic increase in absorption observed as a result of prolonged laser illumination, future study should confirm that a similar effect is observed in hemozoin. It may also be beneficial to investigate intentionally exposing hemozoin and $\beta$-hematin to excessive laser irradiation in order to increase the absorption at shorter wavelengths, potential damage to leukocytes should be taken into consideration. Furthermore, since the TEM images of the irradiated $\beta$-hematin suggest that there is some degree of chemical degradation resulting in a visible physical change from the organized crystalline nanorods of the pristine sample, it may also have detrimental effects on leukocytes post-phagocytocis that could compromise the cells during detection; however, future viability studies should readily confirm or refute that assertion.

\section{Acknowledgments}

We would like to thank the Biological Engineering Department, the Electron Microscopy Core, and the Christopher S. Bond Life Sciences Center of the University of Missouri for the resources used in this study. We would also like to acknowledge the support of the NIH 1R21CA139186-01 grant that predominantly funded this study. Finally, we would like to thank the other members of the Viator lab whose knowledge and support enabled the development and completion of this project.

\section{References}

1. World Malaria Report, 2010. World Health Organization; 2008.

2. Moody A. Rapid diagnostic tests for malaria parasites. Clinical Microbiology Reviews. 2002; 15(1)

3. Wongsrichanalai C, Barcus MJ, Muth S, Sutamihardja A, Wernsdorfer WH. A review of malaria diagnostic tools: microscopy and rapid diagnostic test (RDT). The American Journal of Tropical Medicine and Hygiene. 2007; 77(6 Suppl)

4. Florens L, Washburn MP, Raine JD, Anthony RM, Grainger M, Haynes JD, Moch JK, Muster N, Sacci JB, Tabb DL, et al. A proteomic view of the Plasmodium falciparum life cycle. Nature. 2002; 419(6906) 
5. Olliaro P, Lombardi L, Frigerio S, Basilico N, Taramelli D, Monti D. Phagocytosis of hemozoin (native and synthetic malaria pigment), and Plasmodium falciparum intraerythrocyte-stage parasites by human and mouse phagocytes. Ultrastructural Pathology. 2000; 24(1)

6. Noland GS, Briones N, Sullivan DJ, et al. The shape and size of hemozoin crystals distinguishes diverse Plasmodium species. Molecular and Biochemical Parasitology. 2003; 130(2)

7. Serebrennikova YM, Patel J, Garcia-Rubio LH. Interpretation of the ultraviolet-visible spectra of malaria parasite Plasmodium falciparum. Applied Optics. 2010; 49(2)

8. Lyke KE, Diallo DA, Dicko A, Kone A, Coulibaly D, Guindo A, Cissoko Y, Sangare L, Coulibaly S, Dakouo B, et al. Association of intraleukocytic Plasmodium falciparum malaria pigment with disease severity, clinical manifestations, and prognosis in severe malaria. The American Journal of Tropical Medicine and Hygiene. 2003; 69(3)

9. Custer JR, Kariuki M, Beerntsen BT, Viator JA. Photoacoustic detection of hemozoin in human mononuclear cells as an early indicator of malaria infection. Proceedings of SPIE. 2010; volume 7564

10. Egan T, Mavuso W, Ncokazi K. The mechanism of beta-hematin formation in acetate solution. parallels between hemozoin formation and biomineralization processes. Biochemistry. 2001; 40(1)

11. Tekwani BL, Walker LA. Targeting the hemozoin synthesis pathway for new antimalarial drug discovery: technologies for in vitro beta-hematin formation assay. Combinatorial Chemistry \&\# 38; High Throughput Screening. 2005; 8(1)

12. Cole KA, Ziegler J, Evans CA, Wright DW. Metalloporphyrins inhibit [beta]-hematin (hemozoin) formation. Journal of Inorganic Biochemistry. 2000; 78(2)

13. Rosencwaig, Allan. Photoacoustic spectroscopy. Annual Review of Biophysics and Bioengineering. 1980; 9

14. Viator JA, Jacques SL, Prahl SA. Depth profiling of absorbing soft materials using photoacoustic methods. Selected Topics in Quantum Electronics, IEEE Journal of. 1999; 5(4)

15. Paltauf G, Schmidt-Kloiber H, Guss H. Light distribution measurements in absorbing materials by optical detection of laser-induced stress waves. Applied Physics Letters. 1996; 69(11)

16. Sudduth ASM, Goldschmidt BS, Samson EB, Whiteside PJD, Viator JA. Total internal reflection photoacoustic detection spectroscopy. Proceedings of SPIE. 2010

17. Weight RM, Viator JA, Dale PS, Caldwell CW, Lisle AE. Photoacoustic detection of metastatic melanoma cells in the human circulatory system. Optics Letters. 2006; 31(20)

18. Viator JA, Gupta S, Goldschmidt BS, Bhattacharyya K, Kannan R, Shukla R, Dale PS, Boote E, Katti K. Gold nanoparticle mediated detection of prostate cancer cells using photoacoustic flowmetry with optical reflectance. Journal of Biomedical Nanotechnology. 2010; 6(2)

19. Talbert RJ, Holan SH, Viator JA. Photoacoustic discrimination of viable and thermally coagulated blood using a two-wavelength method for burn injury monitoring. Physics in Medicine and Biology. 2007; 52(7)

20. Weisstein, Eric W. Least squares fitting-exponential. MathWorld - A Wolfram Web Resource. 2012. URL: http://mathworld.wolfram.com/LeastSquaresFittingExponential.html.

21. Orjih AU, Fitch CD. Hemozoin production by plasmodium falciparum: variation with strain and exposure to chloroquine. Biochimica et Biophysica Acta (BBA)-General Subjects. 1993; 1157(2) 


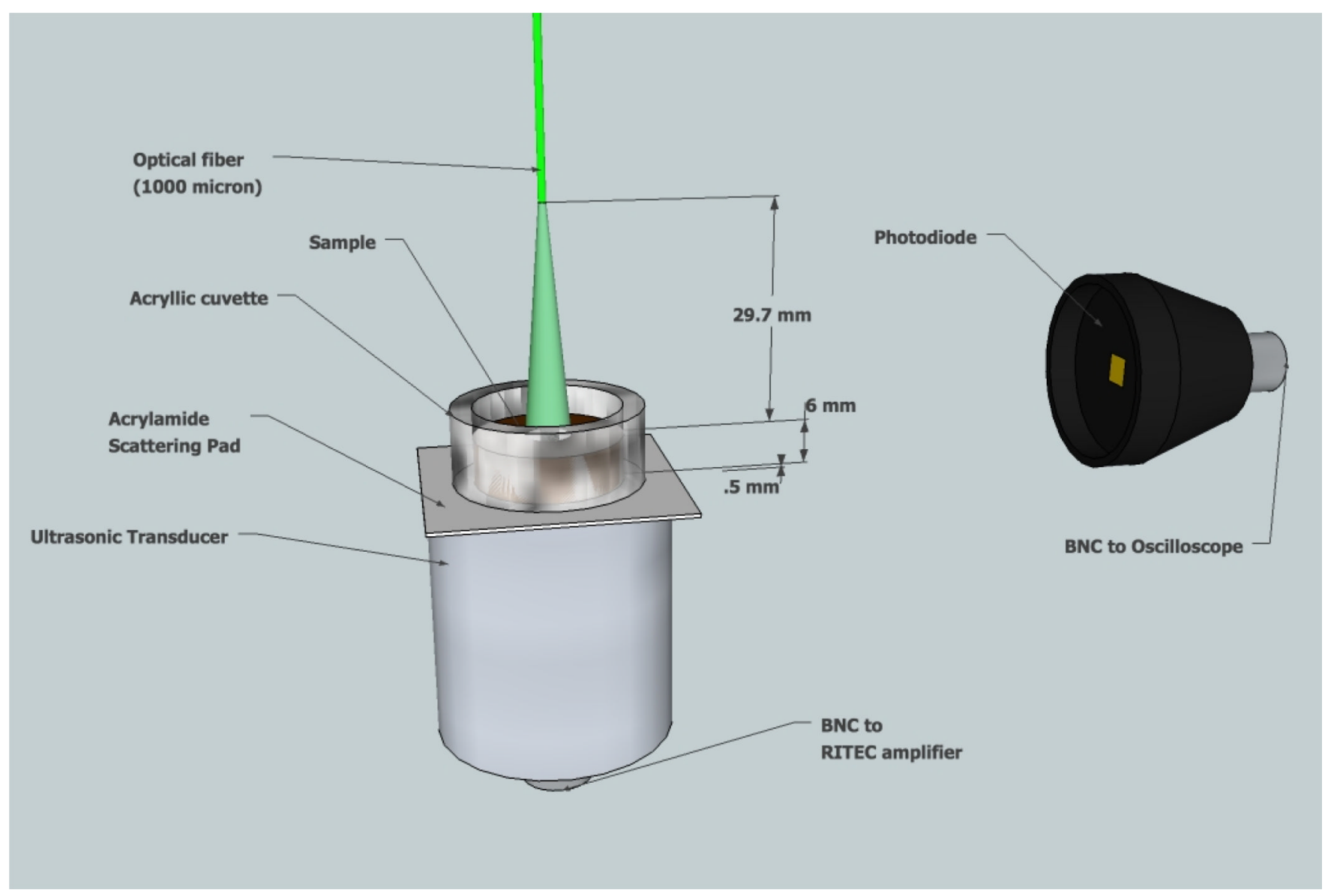

Figure 1.

Conceptual diagram of the photoacoustic apparatus used to record ultrasonic waveforms generated from rapid thermoelastic expansion of $\beta$-hematin following the absorption of pulsed laser light. 


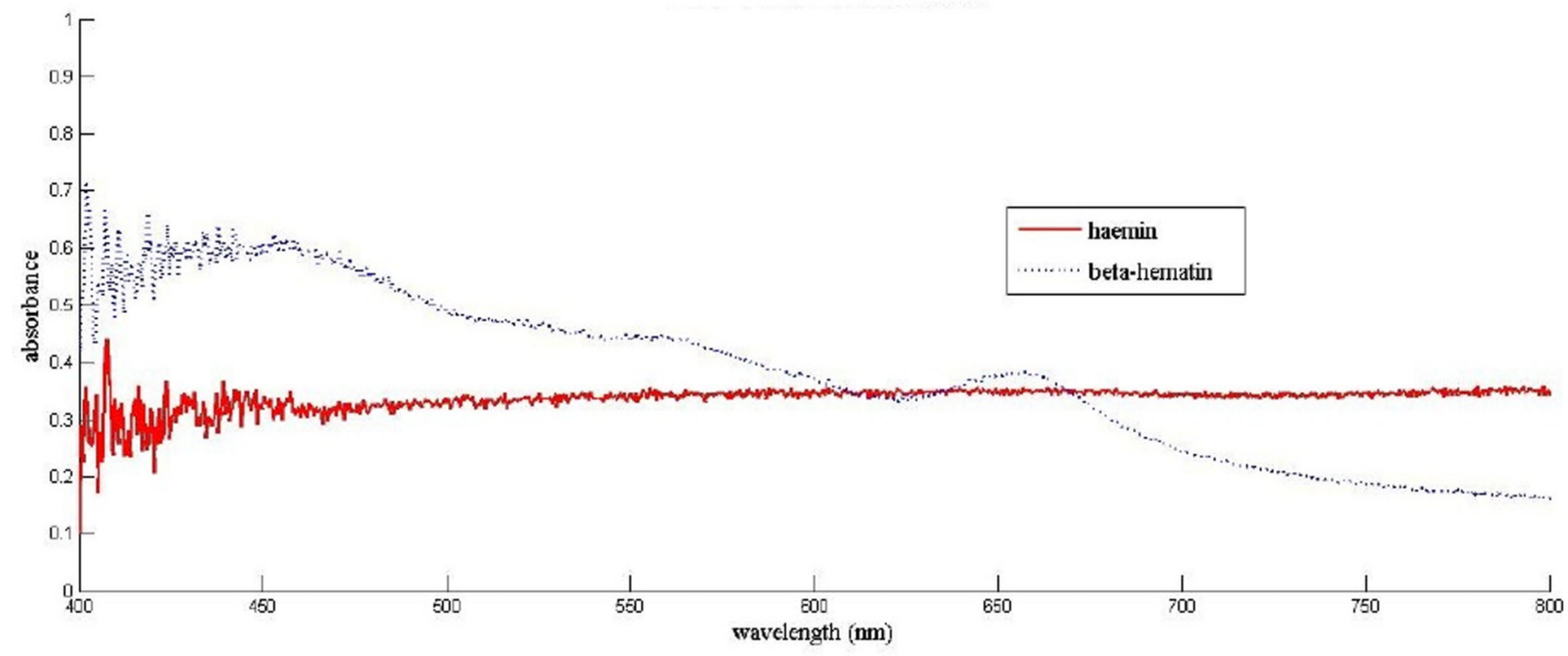

Figure 2.

A comparison of the UV-vis spectroscopy absorbance spectra of $\beta$-hematin and its porcine haemin precursor. 


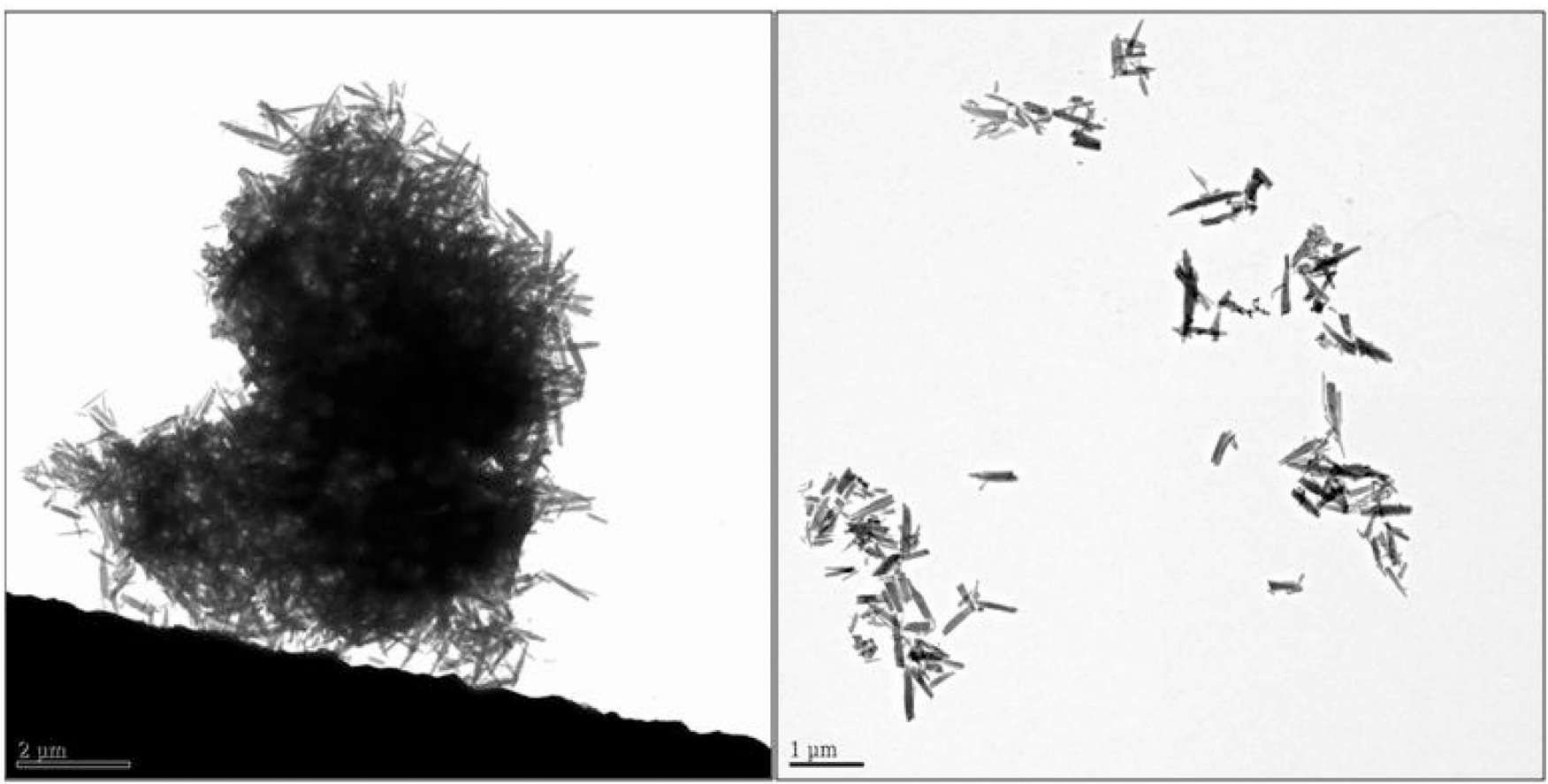

Figure 3.

(Left) TEM image showing a large clumping of $\beta$-hematin due to hydrogen bonding between crystals. (Right) TEM image showing smaller groupings of $\beta$-hematin nanorods. 


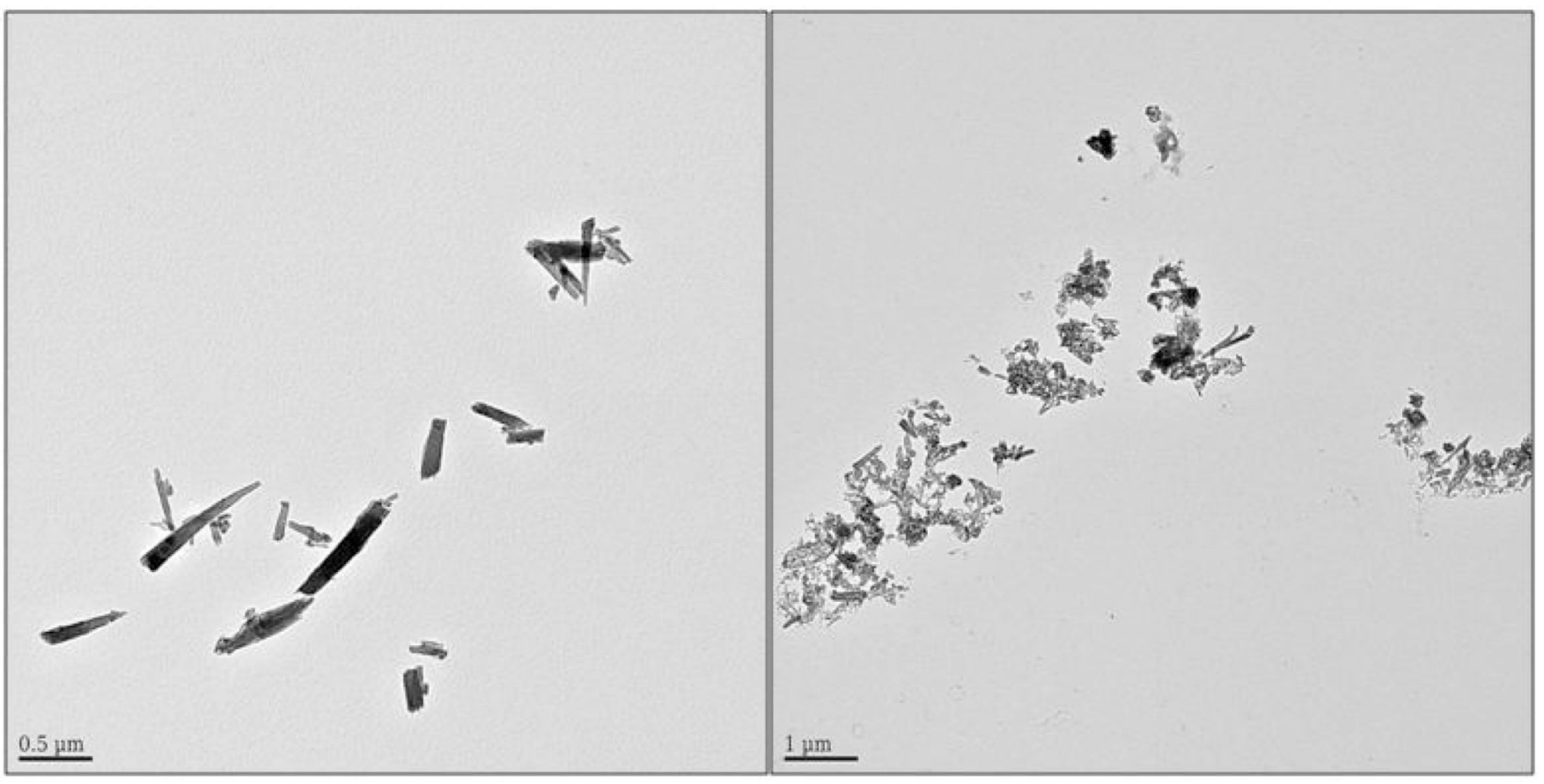

Figure 4.

(Left) TEM image of pristine $\beta$-hematin prior to irradiation. (Right) TEM image of $\beta$ hematin crystals following prolonged exposure to laser irradiation. 


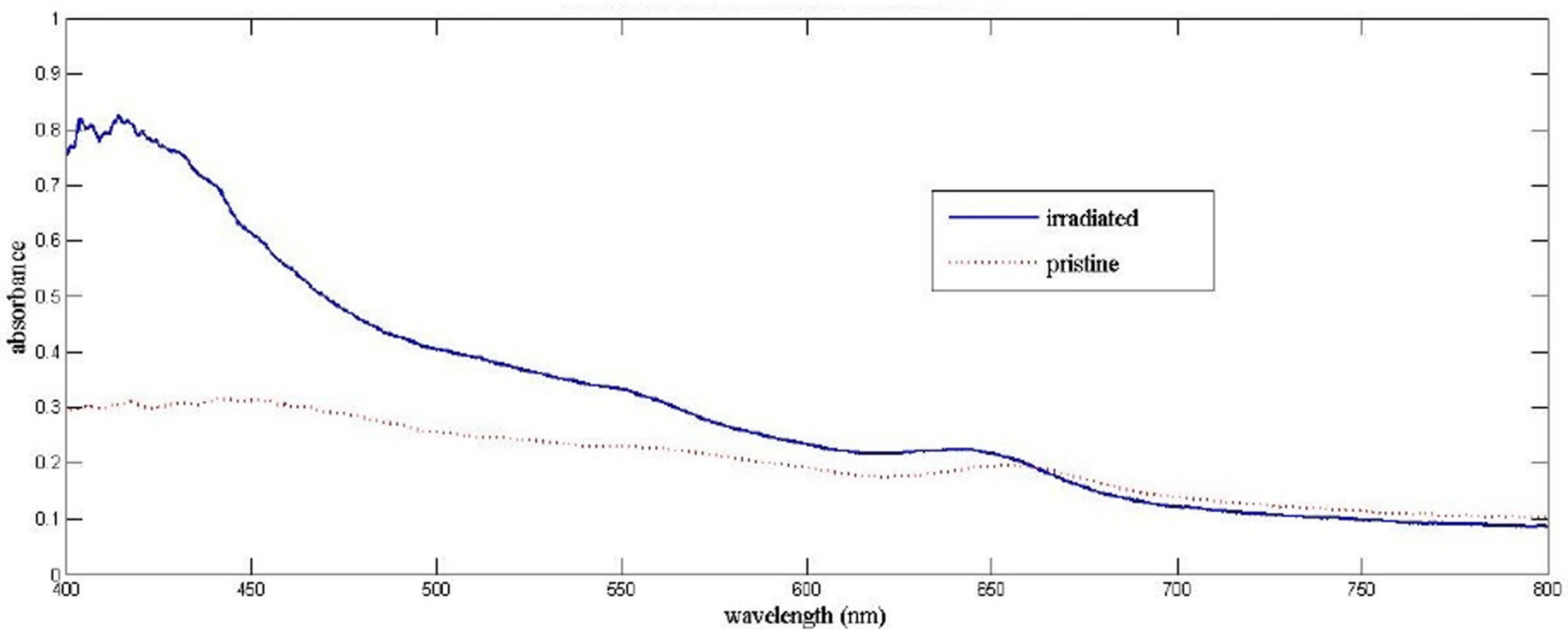

Figure 5.

A comparison of the absorption spectra of dilute $\beta$-hematin suspended in PBS before and after prolonged laser irradiation. 


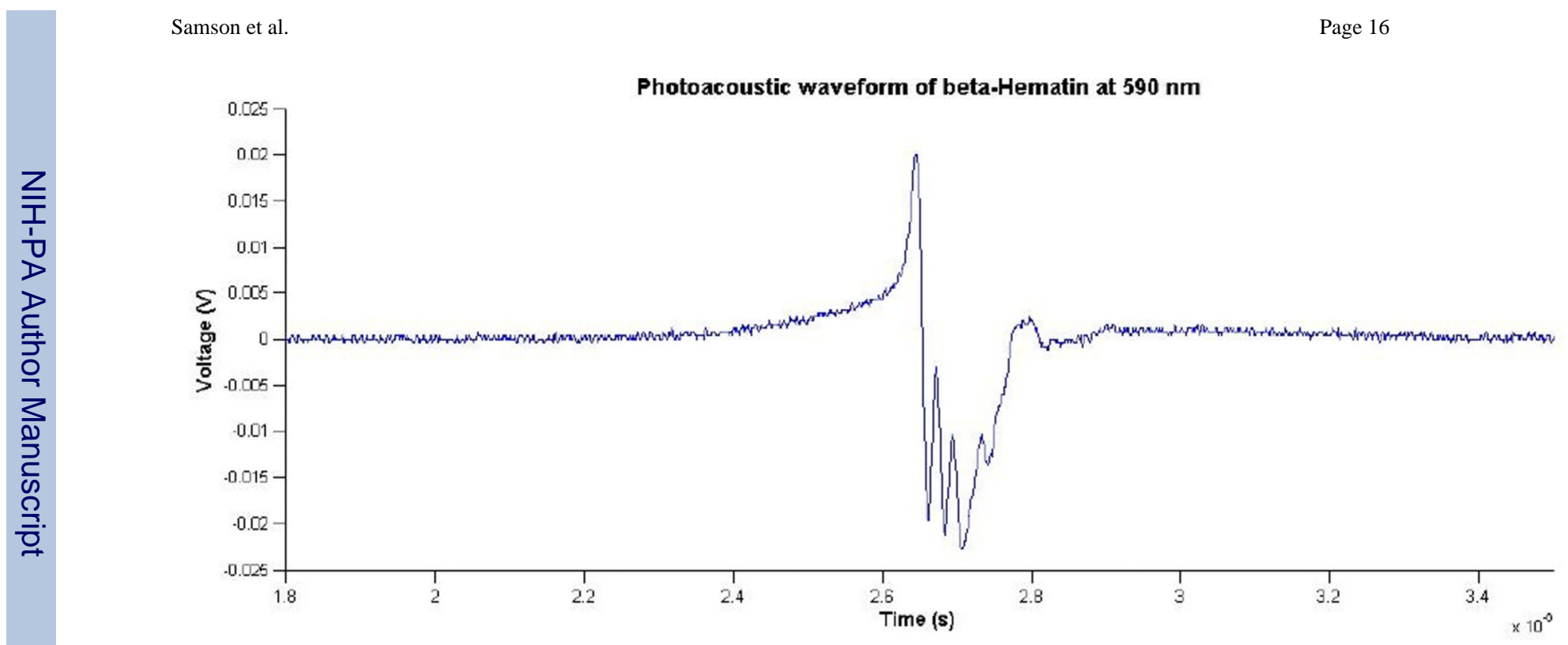

Figure 6.

Sample photoacoustic waveform generated by illumination of $\beta$-hematin by $590 \mathrm{~nm}$ laser light. 

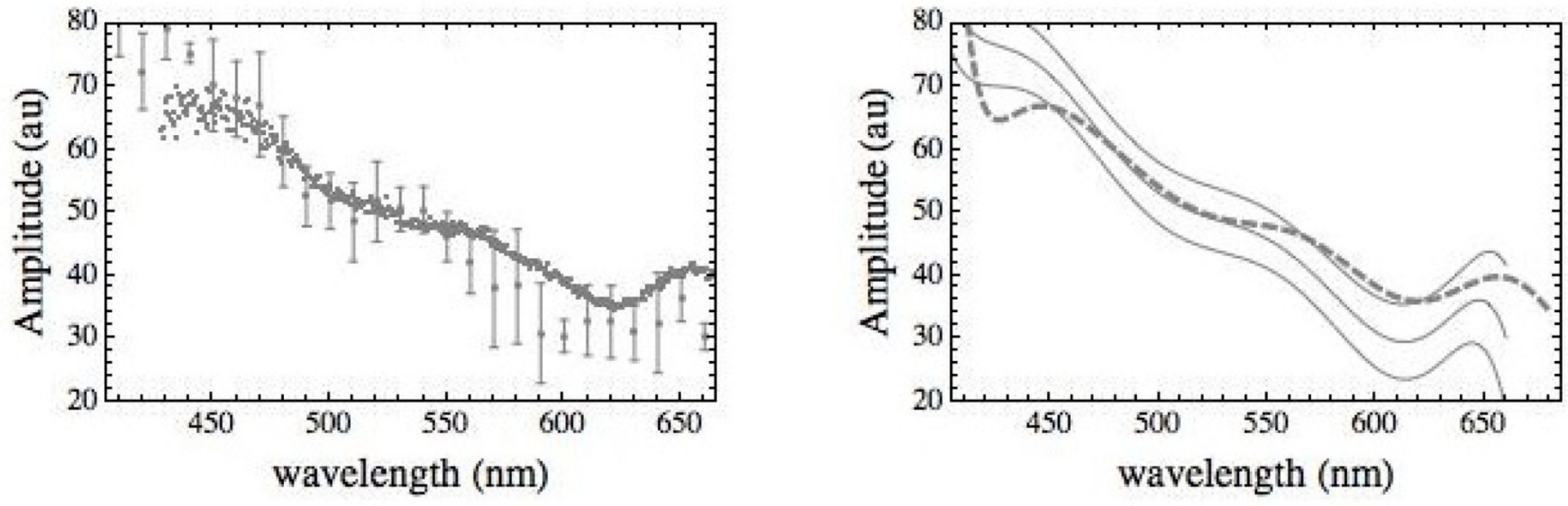

Figure 7.

(Left) A spectrum of calculated absorption coefficients for $1 \frac{m g}{m l} \beta$-hematin suspension derived from photoacoustic waveforms. (Right) Spectra of absorption coefficients derived via UV-vis spectroscopy for the same solution. 


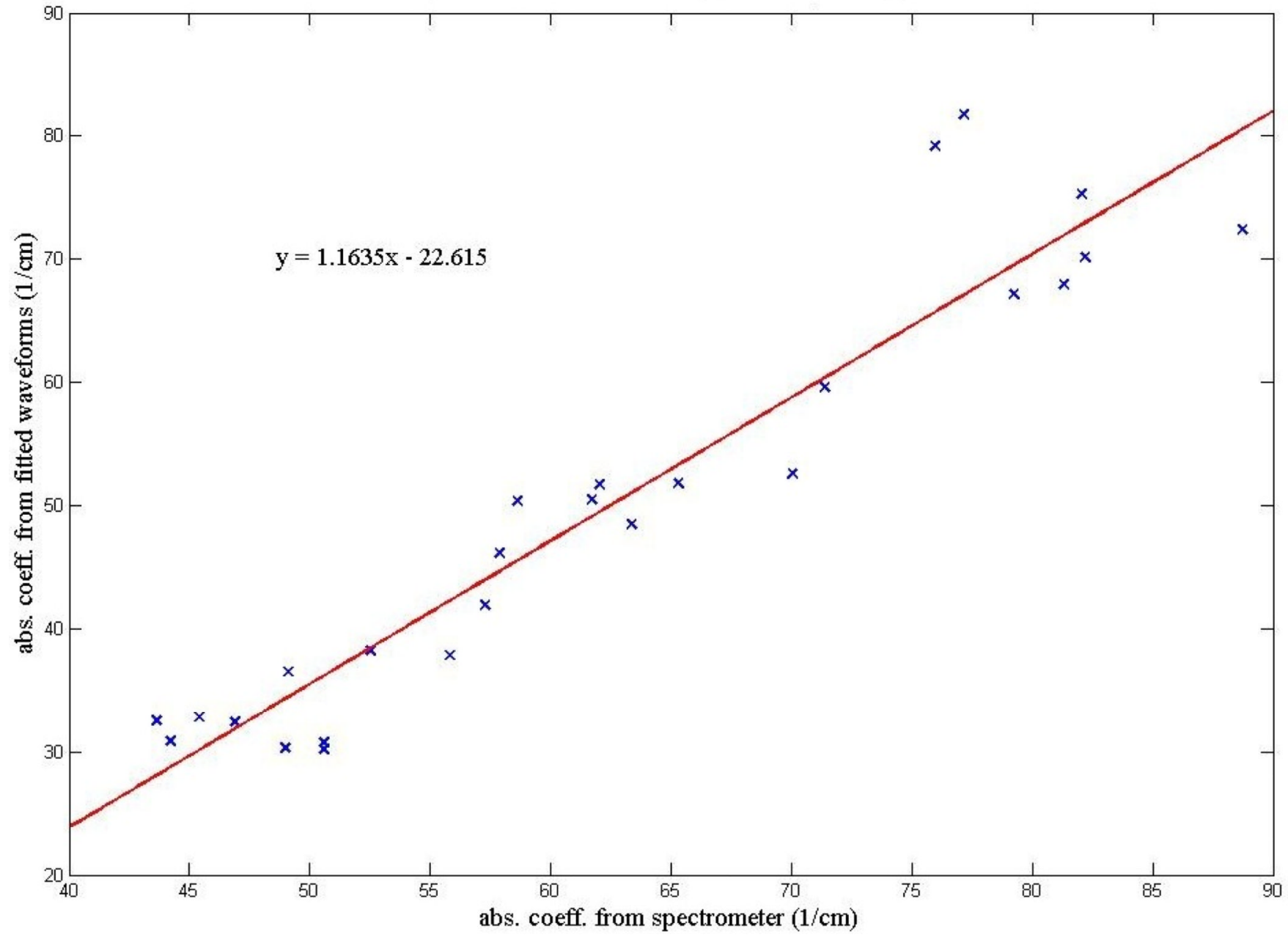

Figure 8.

A graphical relationship of the absorption coefficients derived from UV-vis spectroscopy against those calculated from curve-fitting photoacoustic waveforms 